\title{
Patrones alimentarios y estado nutricional en niños con síndrome de Down en Posadas (Misiones, Argentina)
}

\author{
Eating patterns and nutritional status in children with Down syndrome in \\ Posadas Misiones Argentina
}

\author{
Ornella Vanesa Ghiglione ${ }^{1}{ }^{\circledR}$ \\ orne_vag@hotmail.com
}

\author{
Andrea Roxana López ${ }^{1}$ \\ andrearox99@hotmail.com
}

Artículo recibido: 31/05/2021

Revisado por pares

Artículo aceptado: 08/02/2022

Artículo publicado: 25/02/2022

Autor de correspondencia

Ornella Vanesa Ghiglione,

orne_vag@hotmail.com

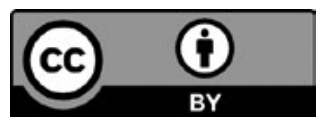

(CLas autoras, 2022. Publicado por la Universidad Norbert Wiener (Lima, Perú)

Citar como: Ghiglione OV, López AR. Patrones alimentarios y estado nutricional en niños con síndrome de Down en Posadas (Misiones, Argentina). Revista de Investigación (de la Universidad Norbert Wiener). 2022;11(1): a0004. doi: https://doi.org/10.37768/unw.rinv.11.01.a0004

\begin{abstract}
Resumen
El presente estudio se desarrolló en diferentes instituciones de educación especial en la ciudad de Posadas (Misiones, Argentina) en el periodo agostoseptiembre del 2017. El objetivo del estudio es evaluar el estado nutricional y los patrones alimentarios en niños de 2 a 12 años con síndrome de Down. El estudio es correlacional de corte transversal. Para la toma de medidas antropométricas, se utilizó una balanza de precisión o báscula de pie y un estadiómetro portátil. Para establecer el patrón alimentario, se realizó una frecuencia de consumo de elaboración propia y el tamizaje de Block. Se incluyeron 30 niños, el 57\% del género masculino y el $43 \%$ del género femenino. Se evidenció, utilizando graficas específicas para síndrome de Down de la Fundación Catalana, que el 63,33\% presenta obesidad; el 53\%, un exceso de peso para la edad; y el $50 \%$ no sigue con un crecimiento lineal normal, e indica talla baja para la edad. En cuanto a la frecuencia de consumo, se identificó un bajo consumo de vegetales y una elevada ingesta de harinas refinadas y alimentos fuente de carbohidratos simples (bebidas azucaradas y postres), grasa saturada y colesterol. El cuestionario de tamizaje de Block para el consumo de grasas mostró que un $46,66 \%$ obtuvo un puntaje mayor a 27 , lo que indica una dieta muy alta en grasas. Para el consumo de verduras, frutas y fibra, un $46,66 \%$ recibió un puntaje menor a 20 , lo que corresponde a una dieta baja en nutrientes importantes. A partir de los resultados obtenidos, se concluye que existe una correlación significativa entre la obesidad y el patrón alimentario predominante.
\end{abstract}

Palabras claves: síndrome de Down, estado nutricional, patrón alimentario

\begin{abstract}
The present study was developed in different special education institutions in the city of Posadas Misiones Argentina during August-September, 2017. The objective of the study is to evaluate the nutritional status and eating patterns in children from two to 12 years of age with Down syndrome. The study is crosssectional and correlational. To obtain anthropometric measurements, we used a precision balance or standing scale and a portable stadiometer. To identify eating patterns, we established a self-reported consumption frequency and used the Block Dietary Fat Screener. Results: Thirty children were included, 57\% were male and $43 \%$ were female. It was evidenced, using specific charts from the Catalan Down syndrome Foundation, that $63.33 \%$ had obesity, 53\% were overweight for their age and 50\% did not comply with normal linear growth, which indicates low height for age. Regarding frequency of consumption, we
\end{abstract}

${ }^{1}$ Instituto Universitario de Ciencias de la Salud, Fundación H. A. Barceló. Santo Tomé, Argentina. 
could determine low consumption of vegetables and a high intake of refined flour and foods which were source of simple carbohydrates (sugary drinks and desserts), saturated fat and cholesterol. The Block Dietary Fat Screener questionnaire for fat consumption indicated that $46.66 \%$ obtained a score higher than 27, which shows a very high-fat diet. In regard to the consumption of vegetables, fruits and fiber, $46.66 \%$ received a score lower than 20 , which corresponds to a diet that is low in important nutrients. Based on the results obtained, it is concluded that there is a significant correlation between obesity and the predominant eating pattern.

Keywords: Down syndrome, nutritional status, feeding behavior

\section{INTRODUCCIÓN}

En la edad infantil, el crecimiento es una característica importante. Son cambios morfológicos y estructurales que modifican la masa corporal, al aumentar el número y el tamaño de las células del organismo.

El crecimiento en los niños con síndrome de Down ocurre de manera diferente. Se distingue por un inicio más temprano y acelerado, pero en años posteriores existe una reducción de la velocidad de desarrollo, lo que genera una estatura más corta que la de la población general. Existe una mayor predisposición al exceso de peso, especialmente entre los adolescentes y adultos, debido a su propia anomalía genética. A su vez, se reducen las necesidades energéticas resultantes por la menor estatura, lo que causa ganancia de peso, por lo que es necesario evaluarlos usando gráficas específicas para esta población y, a su vez, es clave para determinar la presencia de factores asociados con fallas en el crecimiento $\left({ }^{1}\right)$.

Los niños con síndrome de Down constituyen una población endeble, presentan un conjunto de complicaciones que afectan su estado nutricional y su crecimiento y desarrollo. Por ello, su bienestar corporal depende de que estas alteraciones se encuentren controladas $\left({ }^{2}\right)$.

Los niños presentan problemas en la masticacion, deglución, formación del bolo alimentario, succión y diversificación alimentaria, debido a las malformaciones de la boca, el tamaño de la lengua, el retraso en la dentición, la hipotonía de los músculos bucofaciales y la protrusión lingual $\left({ }^{3}\right)$.

El retraso en la erupción dentaria es de origen genético. En los niños con síndrome de Down, la aparición de los dientes temporarios ocurre entre los 14 y 18 meses, el doble de tiempo que en la población general. Por este motivo, se retrasa la diversificación alimentaria $\left({ }^{4}\right)$.

Las alteraciones que presentan en la función de la musculatura orofaríngea ocasionan problemas en la deglución y protrusión lingual; por ende, la selección de formas de preparación y texturas alimentarias será blanda. Es muy importante, durante la primera infancia, fortalecer la función motriz para eludir carencias nutricionales y enfermedades en la edad adulta $\left({ }^{3}\right)$.

Cuando se compara la susceptibilidad a la obesidad y el sobrepeso en el SD con otros grupos de población con deficiencia mental de origen genético, encontramos un problema de salud pública $\left({ }^{5}\right)$.

En las personas con síndrome de Down, la causa de obesidad no solo es una dieta inapropiada y una disminución en el gasto calórico, también se debe a una tasa metabólica en reposo y niveles elevados de leptina, lo que determinaría una resistencia a dicha hormona, menor saciedad y, por lo tanto, mayor ingesta y ganancia de peso secundaria $\left(^{6}\right)$.

Elconocimiento delos riesgosy problemasasociados con el síndrome de Down nos permite saber qué alteraciones pueden aparecer y en qué momentos de la vida del individuo, para prevenir o aliviar los problemas de salud. Es importante un seguimiento general que anteponga los problemas y soluciones por controlar, aportando una coordinación entre las distintas especialidades afectadas en cada individuo y los variados problemas que se pueden presentar a lo largo de la vida $\left(^{7}\right)$.

Esta investigación sobre los patrones alimentarios y el estado nutricional de niños con SD no solo otorga conocimientos sobre la población en estudio y sus características. También nos permite conocer cómo la alimentación repercute en el estado nutricional que tenga el niño, y así determinar qué aspectos 
modificar de la dieta o cómo cambiar alguna conducta considerada equivocada para prevenir futuras enfermedades crónicas de la edad adulta, lo que mejora su calidad de vida.

Por otra parte, será útil como base para realizar futuras investigaciones relacionadas con el tema, ya que otorgará conocimientos sobre del manejo de la alimentación y la evaluación del estado nutricional en niños con SD. Aunque es un tema de interés global, a la vez, es muy poco orientado e informado.

\section{MATERIALES Y MÉTODOS}

\section{Consentimiento informado y autorización}

Para cumplimentar el carácter legal y correspondiente de la recolección de datos, se presentaron notas por escrito a las instituciones correspondientes (ver modelo anexo 1).

A cada participante se le entregó el consentimiento informado, en el cual se solicitaba la autorización para que los niños puedan participar del trabajo de investigación y en el mismo se expresó cuál era la finalidad del estudio (ver modelo anexo 2).

\section{Frecuencia de consumo}

Las encuestas nutricionales constituyen un método directo de valoración nutricional, ponen de manifiesto la conducta alimentaria del individuo y, a su vez, generan información acerca de la ingesta alimentaria de una población.

Para obtener la información sobre la ingesta global en un tiempo determinado y los patrones alimentarios de cada niño, se aplicó un cuestionario de frecuencia de consumo de alimentos dirigida a los padres (ver anexo). Esto permitió relacionar la ingesta de alimentos y el estado nutricional actual, y se pudo obtener datos cuantitativos y cualitativos. Para la obtención de datos, se utilizó una lista de los grupos de alimentos: lácteos; carnes y huevos; vegetales $\mathrm{A}, \mathrm{B}, \mathrm{C}$ y frutas; cereales y sus derivados; legumbres; azúcar y dulces; grasas y aceites; el diseño de la frecuencia de consumo fue dividida en tantas columnas como periodos de ingesta. El beneficio de su utilización es que otorga información sobre la ingesta habitual y no altera los patrones de ingesta usual; asimismo, nos permite alertar sobre posibles alteraciones nutricionales causadas por una dieta inadecuada. Estas encuestas pretenden conocer la cantidad y la calidad de los alimentos ingeridos. Como desventaja de este método se destaca que puede ser impreciso por errores de cálculo al recordar las ingestas pasadas, no se puede utilizar en personas que tengan dietas específicas, la lista de alimentos de consumo habitual puede ser extensa, y nunca puede ser completa e incluir todos los alimentos posibles, por lo que la información solo se refiere a los alimentos que están en la lista. La persona entrevistada puede olvidar voluntaria o involuntariamente ciertos alimentos.

La lista de alimentos se expresará en cantidad de veces por semana que se consume un determinado alimento.

Consumo diario: aquel cuya respuesta sea todos los días

Consumo esporádico: entre 1 y 2 veces por semana Consumo frecuente: entre 3 y 4 veces por semana Consumo muy esporádico: $1 \mathrm{vez}$ al mes

No consume: cuando la respuesta sea nunca $\left({ }^{8}\right)$ (ver modelo anexo 3)

\section{Estado nutricional}

Se puede evaluar a partir de datos e indicadores antropométricos.

\section{a. Datos antropométricos}

Peso corporal. Medición que manifiesta la masa corporal total; incluye fluidos, pero no define compartimentos.

Instrumento de medición. Balanza de precisión o báscula de pie marca Cam para niños mayores a 2 años, con sensibilidad de $100 \mathrm{~g}$.

Técnica de medición. La balanza tiene que estar localizada en una superficie plana horizontal y firme. La persona debe estar de pie, inmóvil, en el centro de la plataforma, con el peso del cuerpo distribuido en forma equitativa entre ambos pies, con los brazos caídos naturalmente a los lados. Puede permanecer con la mínima cantidad de ropa posible, liviana; si esto no es así, luego se descontará la prenda. Se registra el peso hasta los $100 \mathrm{~g}$ completos más próximos al equilibrio del fiel de la balanza. 
Talla (estatura) corporal. Es la altura que presenta un individuo en posición vertical desde el punto más alto de la cabeza hasta los talones en posición de "firmes". Se mide en centímetros $(\mathrm{cm})$.

Instrumento de medición. Estadiómetro portátil marca Seca, cinta métrica de metal flexible e inextensible con $2 \mathrm{~m}$ de longitud, y una escuadra móvil con un ángulo de $90^{\circ}$.

Método instalación del estadiómetro. La pared y el piso debe ser lo más lisos posible, sin zócalo ni grada, no inclinado o sin desnivel. La pared y el piso deben formar un ángulo recto de $90^{\circ}$. Para colocarlo, se requieren dos personas. Una de ellas sitúa el estadiómetro entre el piso y la pared, y forman un ángulo de $90^{\circ}$ sin soltarlo. La otra extiende la cinta métrica del estadiómetro de manera vertical con dirección al techo.

Técnica de medición. El niño deberá estar descalzo y con la menor ropa posible, con ningún tipo de objeto en la cabeza, y con sus talones, nalgas y cabeza en contacto con la superficie vertical. Si es necesario, se requerirá que un asistente sostenga los talones para mantenerlos en contacto con el piso. Las piernas deben estar extendidas, los talones juntos, los hombros relajados y ambos brazos al costado del cuerpo para minimizar la lordosis. La cabeza debe sostenerse de forma tal que el borde inferior de la órbita esté en el mismo plano horizontal que el conducto auditivo externo, para formar así el plano de Frankfurt paralelo al piso. Se le pedirá al niño que realice una inspiración profunda, relaje los hombros y se estire lo más alto posible para la lectura completa. Se debe tener en cuenta que si la marca del estadiómetro se encuentra entre un centímetro y otro hay que anotar el valor que esté más próximo; si está a la mitad, se tomará el del centímetro anterior $\left({ }^{9}\right)$ (ver modelo anexo 4 ).

\section{b. Indicadores antropométricos}

Se utilizo las gráficas de crecimiento específicas para niños con síndrome de Down de la Fundación Catalana. Las mismas son las más adecuadas para la población latina. Dada la importancia de la etnia en el crecimiento, es preciso utilizar curvas de referencia con población de la misma raza $\left({ }^{13}\right)$.
Para realizar la evaluación nutricional se utilizaron los siguientes indicadores:

Peso/Edad. Muestra la masa corporal alcanzada en relación con la edad cronológica. Es un índice compuesto, influenciado por la estatura y el peso relativo.

Talla/Edad. Refleja el crecimiento lineal alcanzado en virtud de la edad cronológica y sus deficiencias se relacionan con alteraciones acumuladas a largo plazo en el estado de salud y nutrición.

Los distintos indicadores permiten comparar, en tablas o curvas específicas, una población de referencia.

A partir de la relación de los parámetros antropométricos y los percentiles establecidos en los gráficos de referencia, se considerará lo siguiente:

Exceso: medición por encima del percentil 95

Normal: medición entre los percentiles 10 y 95

Déficit: medición por debajo del percentil $10\left({ }^{10}\right)$ (ver modelo anexo 5)

\section{Fórmula de Waterlow $\left({ }^{11}\right)$}

Porcentaje de peso para la estatura (P/T)

$\frac{\text { Peso real }}{\text { Peso que debería tener para la estatura }}$ X100

Porcentaje de estatura para la edad (T/E)

$\frac{\text { Estatura real }}{\text { Estatura que debería tener para la edad }}$ X100

Datos personales: Nombre y apellido, Edad y Sexo de los niños.

\section{RESULTADOS}

La muestra estuvo conformada por 30 niños de ambos sexos de las diferentes instituciones, los cuales presentaban un rango de edad entre 2 y 12 años. El promedio fue de 6,5 años con un DS de 3,1 . La mínima corresponde a 2 años y la máxima, a 12 años. Con respecto al sexo hubo predominio del femenino. 


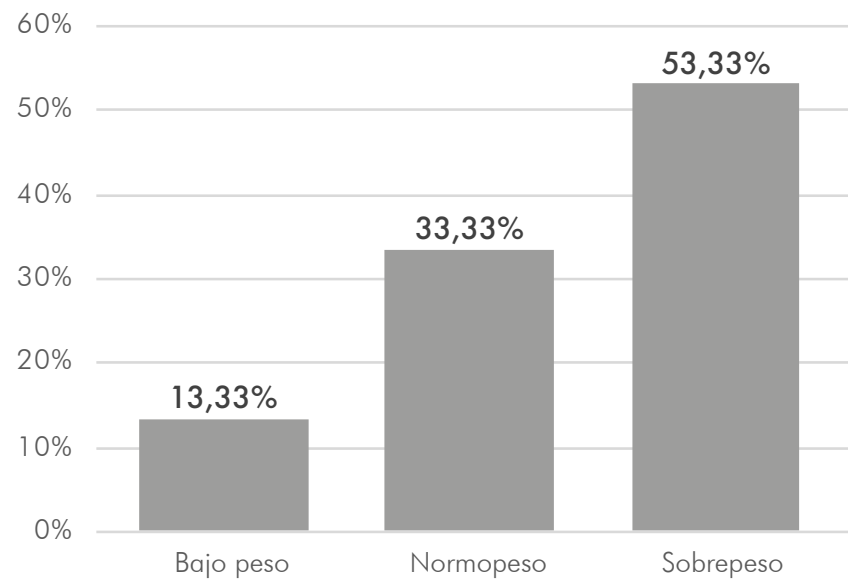

Fuente: Pastor Durán, X. et al. (2004) / Elaboración: Ghiglione, O y López, A. (2017)

Figura 1. Distribución porcentual del estado nutricional de los niños con síndrome de Down que asisten a diferentes instituciones en la ciudad de Posadas (Misiones), según el indicador peso/edad

Se puede observar en la figura 1 que la mayor parte de la población presenta un exceso de peso para la edad.

Se puede observar en figura 2 que la mayoría de la población no sigue un crecimiento lineal normal, pues presentan talla baja para la edad.

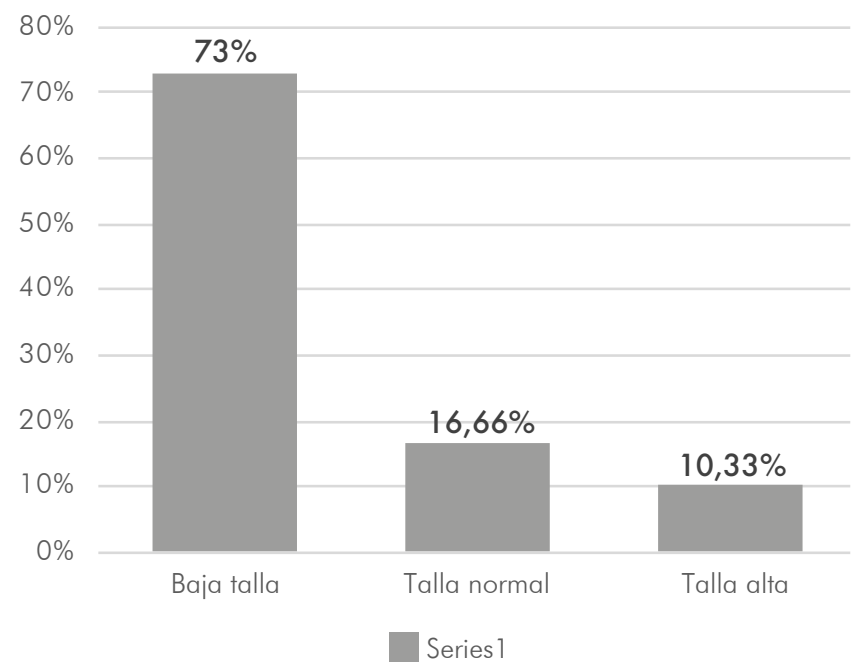

Fuente: Pastor Durán, X. et al. (2004) / Elaboración: Ghiglione, O. y López, A. (2017)

Figura 2. Distribución porcentual de la relación del estado nutricional de los niños con síndrome de Down que asisten a diferentes instituciones en la ciudad de Posadas (Misiones), según el indicador talla/edad

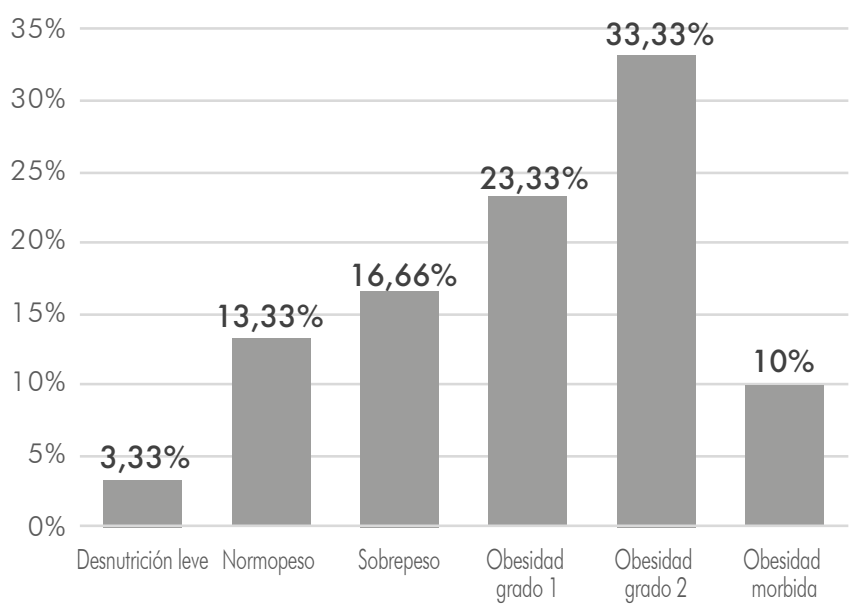

Fuente: Fórmula de Waterlow. / Elaboración: Ghiglione, O. y López, A. (2017)

Figura 3. Distribución porcentual de la relación del estado nutricional de los niños con síndrome de Down que asisten a diferentes instituciones en la ciudad de Posadas (Misiones), según fórmula de Waterlow

En la figura 3 se puede observar que la mayoría de la población presenta un exceso de peso y solo una minoría presenta un peso normal.

En la figura 4 se puede observar que la mayor parte de la población tuvo un puntaje mayor a 27, lo que indica que su dieta es muy alta en grasa.

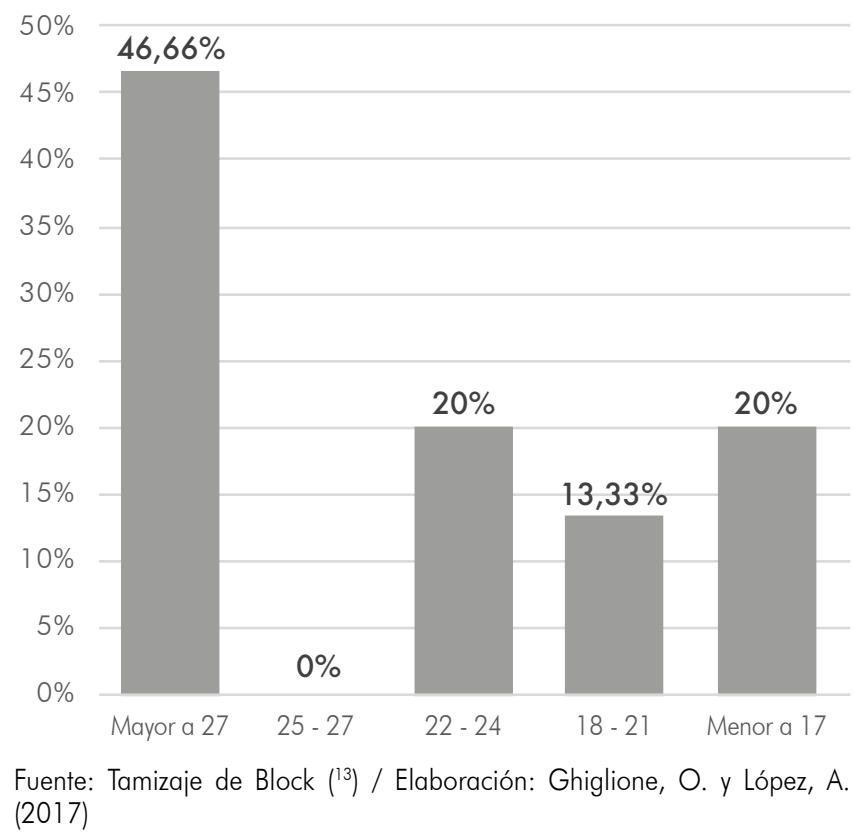

Figura 4. Distribución porcentual de los rangos de puntuación del tamizaje de Block para el consumo de grasas 


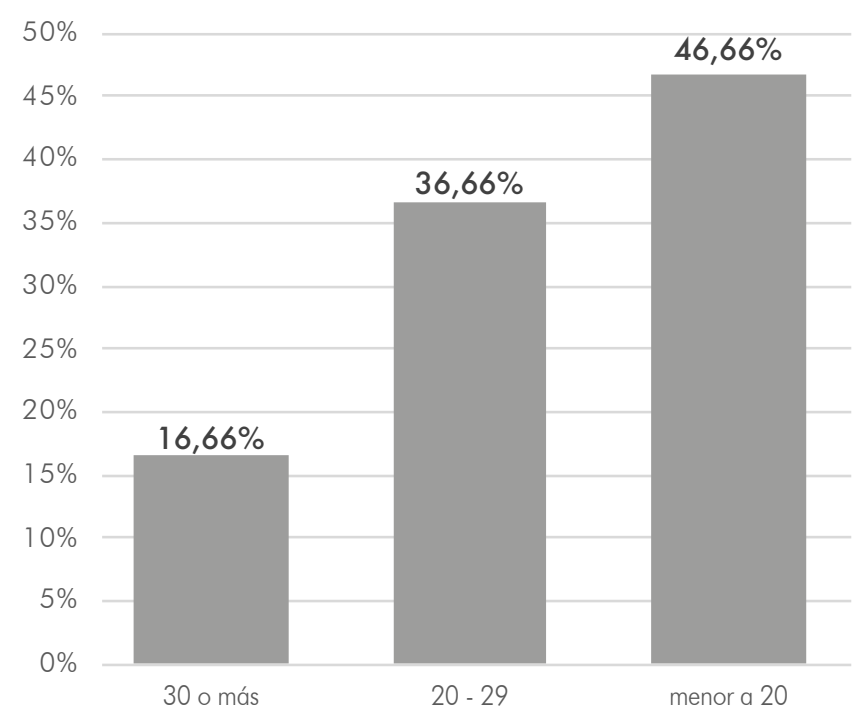

Fuente: Tamizaje de Block / Elaboración: Ghiglione, O. y López, A. (2017)

Figura 5. Distribución porcentual de los rangos de puntuación del tamizaje de Block para el consumo de verduras, frutas y fibra

En la figura 6 se puede observar que la mayoría de la población tiene un puntaje menor a 20, lo que indica que su dieta es baja en nutrientes importantes.

Se observa en la figura 6 que un mayor porcentaje de Waterlow se corresponde con un mayor puntaje del tamizaje, lo que establece una correlación estadísticamente significativa de Pearson, con un $\mathrm{P}$ valor de 0,01 .

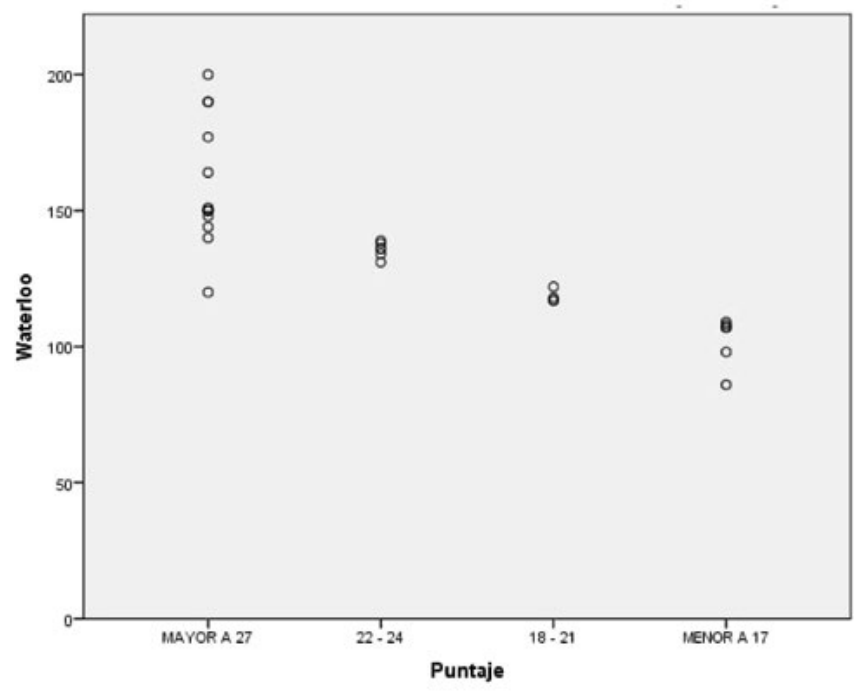

Fuente: Correlación de Pearson / Elaboración: Ghiglione, O. y López, A. (2017)

Figura 6. Lineal de correlación del estado nutricional y tamizaje del Block
Tabla 1. Distribución porcentual de la frecuencia de consumo de cereales en niños con síndrome de Down

\begin{tabular}{lccccc}
\hline \multirow{2}{*}{ Cereales } & \multicolumn{5}{c}{ Frecuencia de consumo } \\
\cline { 2 - 6 } & $\begin{array}{c}\text { Todos los } \\
\text { días }\end{array}$ & $\begin{array}{c}3-4 \text { veces } \\
\text { por semana por semana }\end{array}$ & $\begin{array}{c}1 \text { 1-2 veces al } \\
\text { mes }\end{array}$ & Nunca \\
\hline Cereales & $100 \%$ & $0 \%$ & $0 \%$ & $0 \%$ & $0 \%$ \\
\hline Pan & $66,66 \%$ & $13,33 \%$ & $16,66 \%$ & $3,33 \%$ & $0 \%$ \\
\hline Copos de cereal & $3,33 \%$ & $13,33 \%$ & $43,33 \%$ & $16,66 \%$ & $23 \%$ \\
\hline Galletitas saladas & $16,66 \%$ & $33,33 \%$ & $26,66 \%$ & $6,66 \%$ & $13 \%$ \\
\hline
\end{tabular}

Fuente: Diferentes instituciones de educación especial de la ciudad de Posadas (Misiones). / Elaboración: Ghiglione, O. y López, A. (2017)

Según la tabla 1, el total de la población consume cereales (arroz, fideo, polenta) todos los días, y más de la mitad de la población consume pan diariamente.

En la tabla 2 se observa que, dentro del grupo de los dulces, el alimento más consumido diariamente es el azúcar.

Se puede observar en la tabla 3 que la mayoría de la población consume aceite, manteca y mayonesa diariamente.

Tabla 2. Distribución porcentual de la frecuencia de consumo de dulces en niños con síndrome de Down

\begin{tabular}{lccccc}
\hline \multirow{2}{*}{ Dulces } & \multicolumn{5}{c}{ Frecuencia de consumo } \\
\cline { 2 - 6 } & $\begin{array}{c}\text { Todos los } \\
\text { días }\end{array}$ & $\begin{array}{c}3-4 \text { veces } \\
\text { por semana por semana }\end{array}$ & $\begin{array}{c}1 \text { vez al } \\
\text { mes }\end{array}$ & Nunca \\
\hline Azúcar & $100 \%$ & $0 \%$ & $0 \%$ & $0 \%$ & $0 \%$ \\
\hline Mermelada & $6,66 \%$ & $6,66 \%$ & $43,33 \%$ & $6,66 \%$ & $36,66 \%$ \\
\hline Dulce de leche & $3,33 \%$ & $0 \%$ & $23,33 \%$ & $23,33 \%$ & $50 \%$ \\
\hline Miel & $0,00 \%$ & $10 \%$ & $3,33 \%$ & $26,66 \%$ & $60 \%$ \\
\hline
\end{tabular}

Fuente: Diferentes instituciones de educación especial de la ciudad de Posadas (Misiones). / Elaboración: Ghiglione, O. y López, A. (2017)

Tabla 3.Distribución porcentual de la frecuencia de consumo de grasas en niños con síndrome de Down

\begin{tabular}{lccccc}
\hline \multirow{2}{*}{ grasa } & \multicolumn{5}{c}{ Frecuencia de consumo } \\
\cline { 2 - 6 } & $\begin{array}{c}\text { Todos los } \\
\text { días }\end{array}$ & $\begin{array}{c}3-4 \text { veces } \\
\text { por semana }\end{array}$ & $\begin{array}{c}1-2 \text { veces } \\
\text { por semana }\end{array}$ & $\begin{array}{c}1 \text { vez al } \\
\text { mes }\end{array}$ & Nunca \\
\hline Aceite & $100 \%$ & $0 \%$ & $0 \%$ & $0 \%$ & $0 \%$ \\
\hline Mayonesa & $6,66 \%$ & $6,66 \%$ & $43,33 \%$ & $6,66 \%$ & $36,66 \%$ \\
\hline Manteca & $3,33 \%$ & $0 \%$ & $23,33 \%$ & $23,33 \%$ & $50 \%$ \\
\hline
\end{tabular}

Fuente: Diferentes instituciones de educación especial de la ciudad de Posadas (Misiones). / Elaboración: Ghiglione, O. y López, A. (2017) 


\section{DISCUSIÓN}

Luego de realizado el estudio, cuya finalidad fue determinar el estado nutricional que presentan los niños con síndrome de Down, y la evaluación de los patrones alimentarios, se pudo determinar que el estado nutricional promedio en estos niños fue el de obesidad. Esto se obtuvo mediante la utilización de la fórmula de Waterlow y las curvas de crecimiento para la población de la Fundación Catalana de Síndrome de Down, las cuales son las más apropiadas ya que utilizan una metodología estadística que asegura una mayor exactitud y precisión $\left({ }^{12}\right)$.

Se obtuvo que la mayor parte de la población posee un exceso de peso con respecto al indicador peso/ edad. En cuanto al indicador talla/edad, se halló que la mayor parte de la población no sigue un crecimiento lineal normal y presenta talla baja para la edad.

Con respecto a la evaluación de los patrones alimentarios, se utilizó una frecuencia de consumo alimentario de elaboración propia, la cual nos dio a conocer que la ingesta alimentaria de los niños es alta en alimentos como vegetales $C$, cereales, azúcar, aceites, mayonesa, manteca, golosinas y gaseosas. Para complementar la información, se utilizó el tamizaje de Block, el cual arrojó que el 46,66 \% de la población tuvo un puntaje mayor a 27 , por lo que su dieta es muy alta en grasa. Respecto del consumo de frutas, verduras y fibra, el puntaje fue menor a 20 , lo que indica que su dieta es baja en nutrientes importantes.

La presente investigación tiene similitud en cuanto a los objetivos y los resultados con el estudio de Madrigal y González ( $\left.{ }^{13}\right)$, el cual concluyó que la mitad de la población presentaba un bajo consumo de vegetales y un elevado consumo de harinas refinadas y alimentos fuente de carbohidratos simples, como las golosinas.

El estudio de Álvarez ${ }^{14}$ ) presentó similitud en cuanto al diagnóstico nutricional y el alto consumo de alimentos como cereales, azúcar y grasas. Su objetivo fue establecer la relación entre calidad de la dieta y las medidas antropométricas de niños con síndrome de Down de 6 a 12 años que asisten a un instituto de Ecuador. Los resultados fueron procesados a través del programa EPI INFO versión 2011; la interpretación del IMC se determinó en percentiles y los indicadores talla/edad, en puntaje Z; y se comparó con los valores de referencia de NCHS 2002. De acuerdo con el estado nutricional, el 61,29\% presentaba sobrepeso u obesidad; según la relación talla/edad (T/E), el 74,19\% tenía talla baja severa y el $25,80 \%$, talla baja. A partir de los datos acerca del consumo de alimentos, los alimentos que se ingieren con mayor frecuencia son cereales $(61,29 \%)$, vegetales y legumbres $(58,6 \%)$, frutas frescas $(54,83 \%)$, aceites y grasas $(87,09 \%)$, y azúcar $(93,54 \%)$.

Un estudio realizado en Brasil por Dal Bosco et al. $\left({ }^{15}\right)$ tuvo como objetivo evaluar el estado nutricional de los pacientes con síndrome de Down en la Escuela Especial de Educación del Valle Tacuarí. Para la evaluación antropométrica de individuos de 2 a los 18 años, se utilizaron curvas de crecimiento específicas de Cronk, y se relacionó los indicadores peso/talla. Para individuos de entre 19 y 50 años, se utilizó el índice de masa corporal (OMS, 1998). Se observó similitudes en cuanto a los datos antropométricos y una prevalencia de exceso de peso. En cuanto a la ingesta de alimentos, no se especifica la frecuencia de consumo.

En el estudio realizado por Capurro y Ortiz $\left({ }^{16}\right)$, con el objetivo de determinar el estado nutricional e identificar los hábitos de alimentación en los niños y adolescentes con síndrome de Down que asisten a la Asociación Síndrome de Down de Concepción del Uruguay, se obtuvo, mediante los indicadores peso/talla de las tablas de la Sociedad Argentina de Pediatría (SAP), que del total de la muestra de niños (64\%) y adolescentes (36\%) con síndrome de Down, el 55\% padece obesidad; el 18\%, sobrepeso; el $18 \%$ presenta normopeso; y un $9 \%$, bajo peso. Además, se detectó una prevalencia del $56 \%$ de obesidad infantil, en niños de 3 a 12 años inclusive. Se puede observar similitudes en cuanto a los datos antropométricos, pues hubo una prevalencia de exceso de peso en los niños. En lo que refiere a la calidad alimentaria, la cual se obtuvo a través de una frecuencia de consumo de elaboración propia, se reflejó un consumo excesivo de azúcar, bebidas azucaradas, aceites, carnes y pan. También se registró un consumo inferior de leche, yogur y quesos. 


\section{CONCLUSIONES}

Si bien se conoce que la propia anomalía genética, la baja estatura y el menor requerimiento de necesidades energéticas del individuo con síndrome de Down predisponen al sobrepeso y la obesidad, se pudo observar que el estado nutricional predominante en los niños de 2 a 12 años con este síndrome en diferentes instituciones especiales de Posadas (Misiones, Argentina) es la obesidad. Esto tuvo una relación significativa con el patrón alimentario predominante: un elevado consumo de cereales, dulces y, principalmente, grasas, lo que repercute negativamente en el estado nutricional de los menores. El exceso de nutrientes en la alimentación de los niños con SD respecto del estado nutricional nos muestra que su dieta no es equilibrada y saludable, pues, aunque las cantidades están por encima de lo recomendado, su calidad no es la adecuada.

La mayoría de la población tiene probabilidades de padecer enfermedades cardiovasculares. Esto significa que la ingesta de grasas debe ser controlada, un aspecto a tomar en cuenta, sobre todo, en aquellos niños/as que sufren cardiopatías, las cuales pueden ser controladas mediante el empleo de la dietoterapia.

Observamos valores alarmantes de obesidad en niños con SD, lo que debe llevar a los profesionales de la salud a trabajar de forma conjunta con quienes participan activamente en el proceso de formación del niño, para definir estrategias que inculquen hábitos de vida saludable en edades tempranas, realizar una evaluación nutricional adecuada, fomentar el ejercicio físico y el tratamiento oportuno de comorbilidades.

\section{CONTRIBUCIÓN DE LAS AUTORAS}

Ornella Ghiglione y Andrea López son autoras y responsables de la redacción, revisión y aprobación de la versión final del artículo.

POTENCIALES CONFLICTOS DE INTERESES

Ninguno.

\section{FINANCIAMIENTO}

Este trabajo fue autofinanciado. 


\section{REFERENCIAS BIBLIOGRÁFICAS}

1. Paz MMA. Factores asociados al estado nutricional de niñas y niños con síndrome de Down de 1 a 12 años de la Fundación Margarita Tejada, Guatemala (Tesis de grado). Quetzaltenango: Universidad Rafael Landívar Facultad de Ciencias de la Salud; 2012. Disponible en: http://biblio3.url.edu.gt/ Tesis/2012/09/15/Paz-Michelle.pdf

2. Phuma TE, Mancheno VE. Estado nutricional y hábitos alimentarios en niños de 2 a 5 años que presentan síndrome de Down (Tesis de grado). Quito: Pontificia Universidad Católica de Ecuador; 2015. Disponible en: http://repositorio.puce.edu.ec/ handle/22000/9910

3. González M. Guía de alimentación para personas con sindrome de Down. Madrid: Díaz de Santos; 2014.

4. Federación Iberoamericana de síndrome de Down. Programa Iberoamericano de salud para personas con Sindrome de Down. Madrid: FIADOWN; 2015. Disponible en: https://www.down21-chile.cl/cont/ cont/2015/224_4_programa_iberoamericano_de_ salud.pdf

5. Roizen NJ, Patterson D. Down's syndrome. Lancet. 2003; 361(9365): 1281-9.

6. Elshorbagy AK, Smith AD, Kozich V, Refsum H. Cysteine and obesity. Obesity (Silver Spring). 2012; 20(3): 473-81.

7. Troncoso MV. Síndrome de Down. Influencias de la sociedad. Revista Virtual Síndrome de Down. 2018; $5(200)$.

8. Monsalve JM, González LI. Diseño de un cuestionario de frecuencia para evaluar ingesta alimentaria en la Universidad de Antioquía, Colombia. Nutr Hosp. $2011 ; 26(6)$.

9. De Girolami DH. Fundamentos de valoración nutricional y composición corporal. Buenos Aires: El Ateneo; 2003.

10. Pastor X, Quintó Ll,Corretger M, Gassió R. Tablas de crecimiento actualizadas de los niños españoles con síndrome de Down. Revista Médica Internacional sobre Sindrome de Down. 2004; 3(8).

11. Márquez-González H, García VM, Caltenco ML, García EA, Márquez $\mathrm{H}$, Villa AR. Clasificación y evaluación de la desnutrición en el paciente pediatrico. El Residente. 2012; 7(2): 59-69.
12. Vildoso M. Diagnóstico y manejo nutricional de pacientes con síndrome de Down. Revista Biomédica Revisada Por Pares. 2006; 6(19). Disponible en: www.medwave.cl/link.cgi/medwave/puestadia/ cursos/3519

13. Madrigal A, González AR. Estado nutricional de niños con síndrome Down del Centro Nacional de Educación Especial de Costa Rica. Revista Costarricense de Salud Pública. 2009 Diciembre; 18(2).

14.Álvarez Pichazaca ML. Calidad de la dieta y medidas antropométricas de niños con síndrome de Down de 6 a 12 años, del Instituto Fiscal de Educación Especial Carlos Garbay. Riobamba, 2010-2011 (Tesis de Grado). Riobamba: Escuela Superior Politécnica de Chimborazo; 2011. Disponible en: http://dspace.espoch.edu.ec/ bitstream/123456789/1240/1/34T00218.pdf

15.Dal Bosco SM, Scherer F, Altevogt CG. Estado nutricional de portadores de síndrome de Down no Vale do Taquari - RS. ConScientiae Saúde. 2011; 10(2): 278-84.

16. Capurro YA. Estado nutricional y hábitos alimentarios en niños y adolescentes con síndrome de Down que concurren a la "Asociación Síndrome de Down de Concepción del Uruguay" (A.S.D.C.U)”. Entre Ríos: Universidad de Concepción del Uruguay; 2019. Disponible en: http://repositorio. ucu.edu.ar/jspui/bitstream/522/148/1/TESINA \%20 CAPURRO\%20Y\%20ORTIZ.pdf

17. Block G, Dresser CM, Hartman AM, Carroll MD. Nutrient sources in the American diet: quantitative data from the NHANES II survey. I. Vitamins and minerals. Am J Epidemiol. 1985; 122(1): 1326. Disponible en: https://www.researchgate.net/ publication/19148142_Nutrient_sources_in_the_ American_diet_quantitative_data_from_the NHANES_II_survey_I_Vitamins_and_minerals

18. De Girolami DH. Fundamentos de valoración nutricional y composición corporal. Buenos Aires : El Ateneo; 2004. 